\title{
Usefulness of diffusion tensor imaging in amyotrophic lateral sclerosis: potential biomarker and association with the cognitive profile
}

\author{
Utilidad del tensor de difusión en esclerosis lateral amiotrofica: potencial biomarcador y \\ asociación con el perfil cognitivo
}

Marcelo Chaves', Mariela Bettini', Maria Cecilia Fernandez', Maria Jose Garcia Basalo', Juan Ignacio Rojas', Cristina Besada², Edgardo Cristiano', Angel Golimstok', Marcelo Rugiero'

\begin{abstract}
The objective of this preliminary study was to correlate diffusion tensor imaging (DTI) alterations with the cognitive profile of patients with amyotrophic lateral sclerosis (ALS). Methods: This was a case-control study conducted from December 1, 2012 to December 1, 2014. Clinical and demographic data were recorded. A neuropsychological test battery adapted to ALS patients was used. An MRI with DTI was performed in all patients and fractional anisotropy (FA) was analyzed in the white matter using the tract based spatial statistics program. Results: Twenty-four patients with ALS (15 females, mean age 66.9 + -2.3) and 13 healthy controls (four females, average age $66.9+-2$ ) were included. The DTI showed white matter damage in ALS patients vs. healthy controls $(p<0.001)$. Discussion: In our preliminary study the alterations of white matter in DTI were significantly associated with cognitive impairment in patients with ALS.
\end{abstract}

Keywords: diffusion tensor imaging; amyotrophic lateral sclerosis; biomarkers.

\section{RESUMEN}

El objetivo del presente estudio preliminar fue correlacionar alteraciones del Tensor de Difusión (TD) con el perfil cognitivo de pacientes con Esclerosis Lateral Amiotrofica (ELA). Metodos: Se realizó estudio casos-controles entre el 1 de Diciembre del 2012 hasta el 1 de Diciembre del 2014. Se registraron datos clínicos y demográficos. Se utilizó batería de tests neuropsicológicos adaptada a ELA. Se realizó RMN de cerebro con TD en todos los pacientes, la Fracción de Anisotropía (FA) se analizó en sustancia blanca, utilizando el programa Tract Based Spatial Statistics. Resultados: Se incluyeron 24 pacientes con ELA (15 mujeres, edad media 66.9 + -2.3) y 13 controles sanos (4 mujeres, edad media $66.9+-2$ ). El TD mostró daño en sustancia blanca en los pacientes con ELA vs controles ( $p<0.001$ ). Discusión: En nuestro estudio preliminar las alteraciones de sustancia blanca en TD se asociaron significativamente con alteraciones cognitivas en pacientes con ELA.

Palabras clave: Tensor de difusión; esclerosis lateral amiotrófica; biomarcadores.

Amyotrophic lateral sclerosis (ALS) is a progressive neurodegenerative disease of unknown etiology that is characterized mainly by degeneration of upper and lower motor neurons ${ }^{1}$. Although the motor system is the most affected clinically, it has been reported that up to $50 \%$ of ALS patients have some degree of cognitive impairment in the course of the disease ${ }^{2}$. Striking advances in the understanding of genetics and neuropathology in ALS have built the new concept of a spectrum of the disease that includes both ALS, as well as frontotemporal dementia ${ }^{3}$.

In ALS, the spectrum of cognitive impairment is variable and is expressed only in a subset of affected patients. Therefore, there is currently great interest in developing biomarkers of cognitive and extra-motor systems damage

\footnotetext{
${ }^{1}$ Hospital Italiano de Buenos Aires, Neurology Department, Buenos Aires City, Argentina;

${ }^{2}$ Hospital Italiano de Buenos Aires, Radiology Department, Buenos Aires City, Argentina.

Correspondence: Marcelo Chaves; Neurology Department, Hospital Italiano de Buenos Aires; Peron 4190. Buenos Aires City. Argentina. B1199;

E-mail:pinichaves@hotmail.com

Conflict of interest: There is no conflict of interest to declare.

Received 13 May 2016; Received in final form 19 August 2016; Accepted 22 December 2016.
} 
in ALS. In this regard, new neuroimaging techniques are postulated as potential tools to obtain accurate data and measure early activity in non-motor structures ${ }^{4}$. Diffusion tensor imaging (DTI) is a technique of magnetic resonance imaging (MRI) used to characterize the architecture of the white matter, based on the orientation properties of diffusion of water molecules in the brain ${ }^{5}$. White matter fibers have a preferential orientation of the movement of water molecules, being more restricted in a perpendicular, than in a parallel direction (anisotropic diffusion). The fractional anisotropy (FA) is a measure of the directionality of diffusion, which is reduced when the white matter is injured. Previous studies with DTI in ALS have shown a reduction of FA in the corticospinal tract consistent with the known characteristics of the disease ${ }^{6}$. Recently, studies have shown DTI white matter damage in extra-motor ${ }^{7,8,9,10}$ tracts, but the correlation between the variables of diffusion and clinical presentation have shown inconsistent results to date ${ }^{11}$.

The aim of this preliminary study was to investigate the presence and characteristics of early changes in the DTI in ALS patients and their correlation with the cognitive and clinical profile.

\section{METHODS}

\section{Participants}

A case-control study was performed between December 1, 2012 and December 1, 2014. Patients between 18 and 80 years with a diagnosis of probable or definite ALS according to the El Escorial criteria ${ }^{12}$ were included. Age and sex-matched controls were identified for each ALS patient. These were selected from the ALS patients' relatives or friends. To be included, controls could not present with a cognitive deficit according to the neuropsychological test. There was only one patient and one control who refused participation in the study.

Clinical data was assessed and collected at the Neuromuscular Disease Center of the Hospital Italiano de Buenos Aires. The site of onset, phenotype, age at diagnosis, time to the cognitive assessment and DTI, forced vital capacity, time to gastrostomy, level of physical disability assessed by the ALS revised functional rating scale - (ALSFRS-R) score ${ }^{13}$, and treatment used was recorded.

The present study was carried out with the approval of the Ethics Committee of the Institution and all participants gave informed consent.

\section{Cognitive evaluation}

A battery of neuropsychological tests adapted to patients with ALS (including dysarthria and motor impairment) was used. All neuropsychological tests were performed by neuropsychologists and neurologists of the Cognitive Disorders Center of the Hospital Italiano de Buenos Aires. The neuropsychological test battery included tests used to assess memory (Rey Auditory Verbal Learning Test); executive functions (direct and reverse digit number-letter; Trail Making Test: Motor Speed, Visual Scanning, Number Sequencing, and Letter Sequencing); social cognition (Key Search - BADS, Eye Task Baron Cohen); visuospatial functions (block building); and language (vocabulary - abbreviated Boston Naming Test, phonemic fluency, semantic fluency). In addition, all patients were evaluated by the Addenbrooke's Cognitive Examination revised test ${ }^{14}$ to determine cognitive deficit. A value below 86 points was considered a cognitive deficit for patients with a high education level (complete schooling; more than 12 years of study). A score below 68 points was considered to be a cognitive deficit for patients with low educational level (incomplete schooling). Revised diagnostic criteria were applied for the diagnosis of the behavioral variant of frontotemporal dementia ${ }^{15}$. The diagnosis of other dementias was based on the DSM-IV and NINCDS-ADRDA ${ }^{16}$. Phukan criteria were used for categorizing cognitive deficit in patients without dementia ${ }^{17}$.

\section{Diffusion tensor imaging}

Brain MRI was performed using a 1.5-T Sigma resonator scanner (Avanto Siemens). The sequences obtained were conventional (T1-weighted, proton density and T2-weighted sequences). The DTI sequences were obtained to evaluate the change in white matter tracts. The following parameters were used: TR between 5000 and $8000 \mathrm{~ms}$, TE $66 \mathrm{~ms}, 45$ cuts, resolution of $2 \mathrm{~mm} \times 2 \mathrm{~mm} \times 4 \mathrm{~mm}$. The DT images were corrected for the movement, using a nonlinear technique provided by FMRIB Software Library (FSL). The FA was measured by the tract based spatial statistics software. Spatial reorientation of FA was allowed by the tract based spatial statistics in a standard space with no systematic effect of spatial transformation of the direction of white matter fibers and without the need to select areas of the fibers that could skew the analysis ${ }^{18}$. Data provided by the tract based spatial statistics were analyzed by FA maps using a linear model. The FA was compared between patients and controls and between patients with ALS with and without cognitive impairment, adjusting for clinical and demographic variables. For this analysis, the statistical significance was set at $\mathrm{p}<0.05$.

\section{Statistical analysis}

Baseline characteristics of the cohort were reported as percentages for categorical data and media with its standard deviation for continuous data. Data were compared using Fisher's exact test and the Mann Whitney U test for categorical and continuous data respectively. Data from FA maps were correlated with clinical findings by a regression model. The collection and data analysis were performed using STATA 10.1 program. 


\section{RESULTS}

A total of 24 patients with sporadic ALS and 13 healthy controls (11 ALS friends and two ALS relatives) were included. Demographic and clinical characteristics of patients with ALS and controls are shown in the Table. The most frequent cognitive profile was the multi-domain executive dysfunction ( $36 \%, \mathrm{n}=10)$. The DTI showed diffuse damage of the white matter in patients with ALS (Figure 1). The greatest alteration was observed in the bilateral frontal and parietal white matter.

Multivariate logistic regression analysis showed that the mean value of FA was lower in ALS patients vs. healthy controls $(\mathrm{p}<0.001)$, (Table). The greatest damage to white matter was observed in patients with cognitive impairment (Figure 2). The multi-domain executive dysfunction profile was associated with significantly increased white matter damage, after multivariate analysis $(\mathrm{p}<0.001)$ (Table).

It was also observed that patients with ALS and cognitive impairment showed greater involvement of diffuse white matter bilaterally vs. ALS patients without cognitive dysfunction. This difference was significant $(p<0.001)$, after logistic regression analysis,

\section{DISCUSSION}

Our preliminary study showed a diffuse alteration of the cerebral white matter, measured by DTI in sporadic ALS patients compared to healthy controls. It was observed that the greater involvement of white matter (lower FA values in DTI) was significantly associated with the presence of cognitive deficits. These findings support previous studies with voxel-based morphometry, which observed that ALS patients showed greater cognitive dysfunction and atrophy in extra-motor regions ${ }^{19}$.

Recently, diverse authors have reported an association between alterations in DT images and disease severity in patients with ALS ${ }^{6,10}$. However DTI longitudinal studies in ALS patients have yielded conflicting results regarding the correlation of progression of brain damage and concomitant worsening of disability ${ }^{20}$. In our series of patients with ALS, no association between the degree of disability, as assessed by the ALSFRS-R scale, and alterations in the DTI was found.

Few studies have evaluated the early neuroimaging findings in ALS patients with mild neuropsychological alteration. The imaging correlation of cognitive impairment in ALS has been described more frequently in patients who have ALS associated with frontotemporal dementia, reporting atrophy of the frontal lobe, temporal and frontal hypometabolism reduced perfusion of the frontotemporal cortex and anterior cingulate gyrus ${ }^{21}$. Even less evidence exists of the imaging alterations observed in non-demented patients with ALS but with mild cognitive impairment. Recently, Agosta et al, in a study with DTI, demonstrated the association of the degeneration of white matter tracts with neuropsychological deficits in patients with $\mathrm{ALS}^{22}$.

In our study, we highlight that all patients were evaluated at an early stage of the disease with a full battery of neuropsychological tests adapted to ALS patients, which included all cognitive areas. Phukan criteria, a useful validated cognitive

Table. Baseline characteristics and multivariate logistic regression analysis between ALS patients and controls.

\begin{tabular}{|c|c|c|c|}
\hline Variable & ALS patients $(n=24)$ & Control group $(n=13)$ & p-value \\
\hline Age, years & $66.9 \pm 2$ & $66.9 \pm 2.3$ & 0.99 \\
\hline Female sex, n (\%) & $15(62.5)$ & $4(36.3)$ & 0.15 \\
\hline Age at ALS onset, years & $64.6 \pm 4$ & - & - \\
\hline Disease duration, months & $14.6 \pm 18$ & - & - \\
\hline Bulbar onset, n (\%) & $4(16.7)$ & - & - \\
\hline Spinal onset, n (\%) & 20 (83.3) & - & - \\
\hline Mean ALS FRS-R at analysis, points & $35.2 \pm 8$ & - & - \\
\hline Riluzol at analysis & $15(65.2)$ & - & - \\
\hline Gastrostomy at analysis & 0 & - & - \\
\hline Education, years & $11 \pm 1$ & $10.2 \pm 1$ & 0.56 \\
\hline ALS family history, $\mathrm{n}$ & 0 & 0 & - \\
\hline Dementia family history, n (\%) & $2(8.3)$ & 0 & 0.46 \\
\hline Parkinson's disease family history, n (\%) & $1(4.17)$ & 0 & 0.64 \\
\hline Cognitive dysfunction, $\mathrm{n}(\%)$ & $17(68)$ & 0 & $<0.001$ \\
\hline Multi-domain executive dysfunction, $\mathrm{n}(\%)$ & $10(42)$ & 0 & $<0.001$ \\
\hline Single-domain executive dysfunction, n (\%) & $6(25)$ & 0 & 0.02 \\
\hline Multi-domain non-executive dysfunction, n (\%) & 0 & 0 & - \\
\hline Single-domain non-executive dysfunction, n (\%) & 0 & 0 & - \\
\hline DTI alteration, FA mean value & $0.713 \pm 0.021$ & $0.654 \pm 0.024$ & $<0.001$ \\
\hline
\end{tabular}

ALS: amyotrophic lateral sclerosis; FRS-R: functional rating scale - revised; DTI: diffusion tensor imaging; FA: fractional anisotropy. 

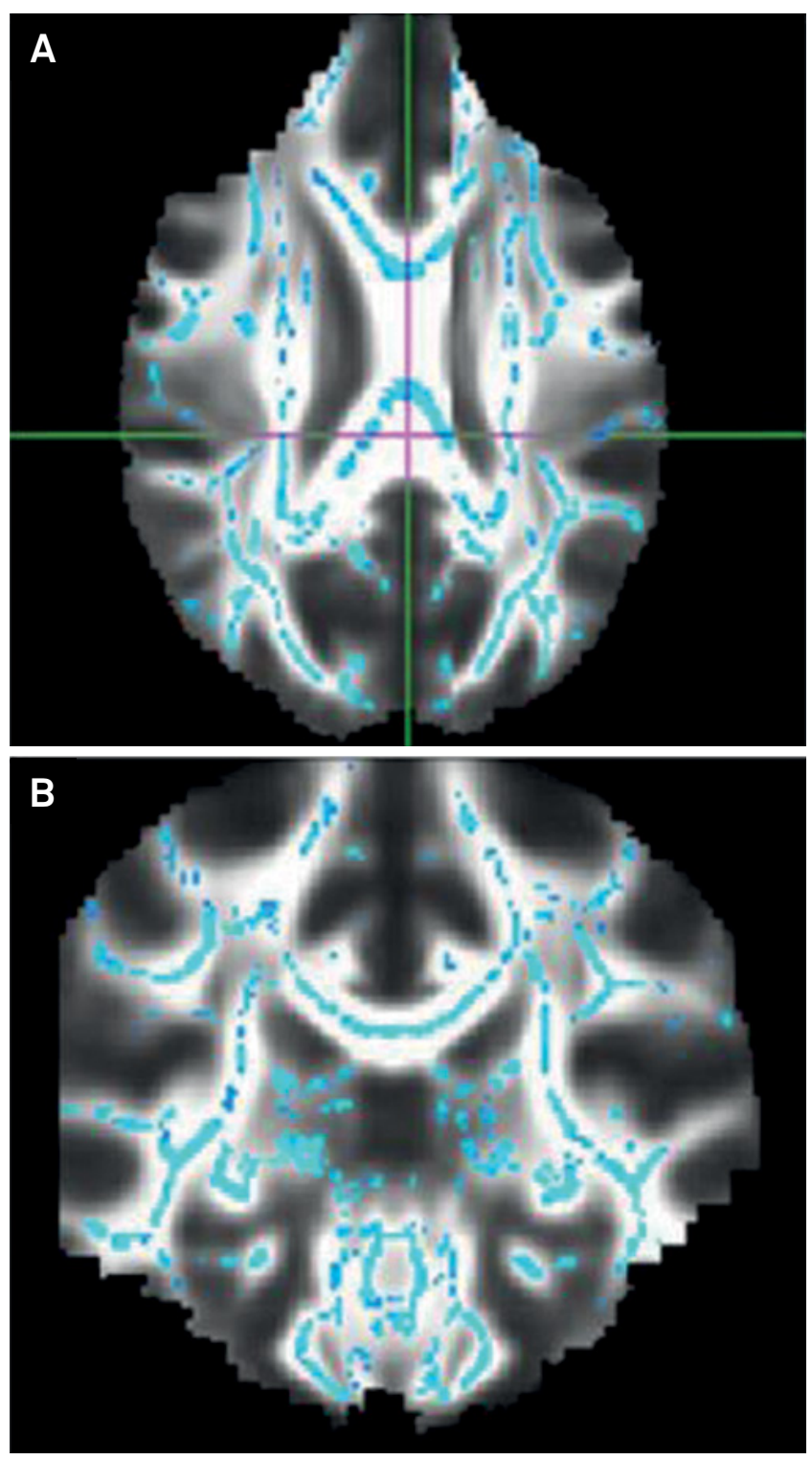

Figure 1. DTI showed diffuse damage of the white matter in patients with ALS

classification for ALS patients, were used for evaluating subtle cognitive impairment in non-demented patients, which is very important in the early stages of the disease. It is relevant to note that these criteria comprehensively address the commitment of various cognitive areas and not just the already-recognized frontal-executive dysfunction described in ALS patients. Although no patients met the criteria for frontotemporal dementia or other dementias, $66 \%$ had some degree of cognitive dysfunction, which is consistent with a recently-published study ${ }^{23}$. The most frequent cognitive profile was the multi-domain executive dysfunction, and it was this subgroup of patients who showed greater impairment of white matter in DTI, showing a pattern of diffuse involvement, predominantly in the frontal lobes.

The neuropsychological profile observed in our group of ALS patients agrees with different reported series. The frontal executive skills deficits, evidenced by impaired sustained attention, working memory, concentration and speed of information
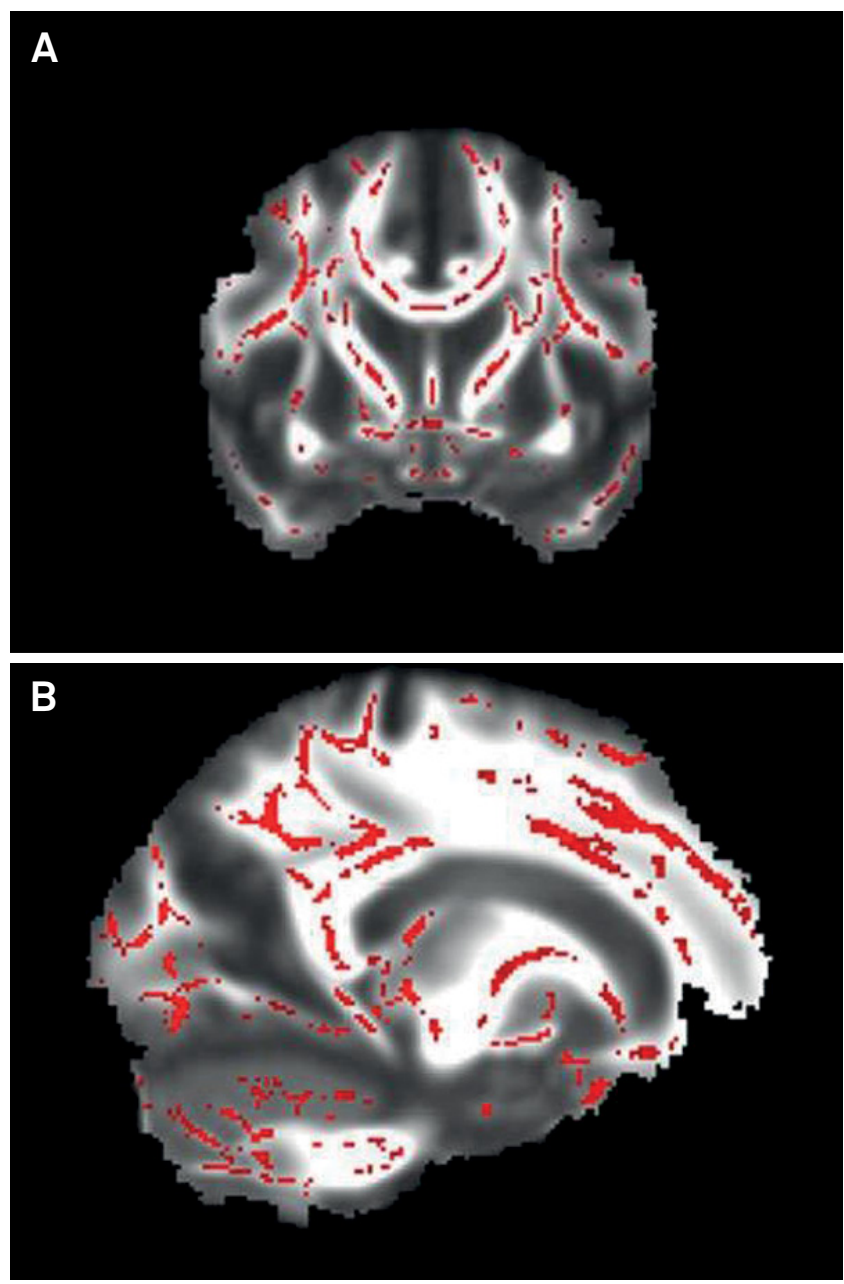

Figure 2. The red shows increase in FA in patients with ALS and without cognitive impairment $(A)$ compared with patients with ALS and cognitive impairment (B).

processing predominated. Furthermore, comparing the subset of ALS patients with cognitive dysfunction versus those without cognitive dysfunction, it was observed that the former showed greater alteration of diffuse white matter in DTI, although it was a minor difference. The latter supports the usefulness of DTI as a tool for objective measurement of impairment of extra-motor structures in ALS patients in the early stages of the disease.

A limitation of our study is that genetic testing was not performed to detect mutation in the C9orf72 gene in the patients who were included. This is relevant because patients with this mutation have early cognitive manifestations ${ }^{6}$. We believe it would be useful to assess the changes of DTI in patients with mutations in C9orf72 and cognitive dysfunction. Thus, evaluation with DTI may be useful to characterize and select the sporadic ALS patients requiring such genetic evaluation.

Considering our preliminary findings, DTI would be a very useful tool to characterize accurately and early on, the degree and extent of involvement of white matter in patients with ALS.

Longitudinal studies are needed to confirm this hypothesis and clarify whether DTI can serve as an effective biomarker to predict ALS patients will progress to develop dementia. 
1. Carvalho M, Swash M. Amyotrophic lateral sclerosis: an update. Curr Opin Neurol. 2011;24(5):497-50. https://doi.org/10.1097/WC0.0b013e32834916a9

2. Ringholz GM, Appel SH, Bradshaw M, Cooke NA, Mosnik DM, Schulz PE. Prevalence and patterns of cognitive impairment in sporadic ALS. Neurology. 2005;65(4):586-90. https://doi.org/10.1212/01.wnl.0000172911.39167.b6

3. Ferrari R, Kapogiannis D, Huey ED, Momeni P. FTD and ALS: a tale of two diseases. Curr Alzheimer Res. 2011;8(3):273-94 https://doi.org/10.2174/156720511795563700

4. Rocha AJ, Maia Júnior AC. Is magnetic resonance imaging a plausible biomarker for upper motor neuron degeneration in amyotrophic lateral sclerosis/primary lateral sclerosis or merely a useful paraclinical tool to exclude mimic syndromes? A critical review of imaging applicability in clinical routine. Arq Neuropsiquiatr. 2012;70(7):532-9. https://doi.org/10.1590/S0004-282X2012000700012

5. Basser PJ, Mattiello J, LeBihan D. Estimation of the effective self-diffusion tensor from the NMR spin echo.J Magn Reson B. 1994:103(3):247-54. https://doi.org/10.1006/jmrb.1994.1037

6. Ellis CM, Simmons A, Jones DK, Bland J, Dawson JM, Horsfield MA et al. Diffusion tensor MRI assesses corticospinal tract damage in ALS. Neurology. 1999;53(5):1051-8. https://doi.org/10.1212/WNL.53.5.1051

7. Blain CR, Williams VC, Johnston C, Stanton BR, Ganesalingam J, Jarosz JM et al. A longitudinal study of diffusion tensor MRI in ALS. Amyotroph Lateral Scler. 2007;8(6):348-55. https://doi.org/10.1080/17482960701548139

8. Sach M, Winkler G, Glauche V, Liepert J, Heimbach B, Koch MA et al. Diffusion tensor MRI of early upper motor neuron involvement in amyotrophic lateral sclerosis. Brain. 2004;127(2):340-50. https://doi.org/10.1093/brain/awh041

9. Chen Z, Ma L. Grey matter volume changes over the whole brain in amyotrophic lateral sclerosis: a voxel-wise meta-analysis of voxel based morphometry studies. Amyotroph Lateral Scler. 2010;11(6)549-54. https://doi.org/10.3109/17482968.2010.516265

10. Agosta F, Pagani E, Rocca MA, Caputo D, Perini M, Salvi F et al. Voxel-based morphometry study of brain volumetry and diffusivity in amyotrophic lateral sclerosis patients with mild disability. Hum Brain Mapp. 2007;28(12):1430-8. https://doi.org/10.1002/hbm.20364

11. Cosottini M, Giannelli M, Siciliano G, Lazzarotti G, Michelassi MC, Del Corona A et al. Diffusion-tensor MR imaging of corticospinal tract in amyotrophic lateral sclerosis and progressive muscular atrophy. Radiology. 2005;237(1):258-64. https://doi.org/10.1148/radiol.2371041506

12. Brooks BR. El Escorial World Federation of Neurology criteria for the diagnosis of amyotrophic lateral sclerosis. Subcommittee on Motor Neuron Diseases/Amyotrophic Lateral Sclerosis of the World Federation of Neurology Research Group on Neuromuscular
Diseases and the El Escorial "Clinical limits of amyotrophic lateral sclerosis" workshop contributors. J Neurol Sci. 1994;124 Suppl:96-107. https://doi.org/10.1016/0022-510X(94)90191-0

13. The Amyotrophic Lateral Sclerosis Functional Rating Scale. Assessment of activities of daily living in patients with amyotrophic lateral sclerosis. The ALS CNTF treatment study (ACTS) phase I-II Study Group. Arch Neurol. 1996;53(2):141-7. https://doi.org/10.1001/archneur.1996.00550020045014

14. Mioshi E, Dawson K, Mitchell J, Arnold R, Hodges JR. The Addenbrooke's Cognitive Examination Revised (ACE-R): a brief cognitive test battery for dementia screening. Int J Geriatr Psychiatry. 2006;21(11):1078-85. https://doi.org/10.1002/gps.1610

15. Rascovsky K, Hodges JR, Knopman D, Mendez MF, Kramer JH, Neuhaus $J$ et al. Sensitivity of revised diagnostic criteria for the behavioural variant of frontotemporal dementia. Brain. 2011;134(9):2456-77. https://doi.org/10.1093/brain/awr179

16. McKhann G, Drachman D, Folstein M, Katzman R, Price D, Stadlan EM Clinical diagnosis of Alzheimer's disease: report of the NINCDS-ADRDA Work Group under the auspices of Department of Health and Human Services Task Force on Alzheimer's Disease. Neurology. 1984;34(7):939-44. https://doi.org/10.1212/WNL.34.7.939

17. Phukan J, Elamin M, Bede P, Jordan N, Gallagher L, Byrne S et al. The syndrome of cognitive impairment in amyotrophic lateral sclerosis: a population-based study. J Neurol Neurosurg Psychiatry. 2012;83(1):102-8. https://doi.org/10.1136/jnnp-2011-300188

18. Smith SM. Fast robust automated brain extraction. Hum Brain Mapp. 2002;17(3):143-55. https://doi.org/10.1002/hbm.10062

19. Abrahams S, Goldstein LH, Suckling J, Ng V, Simmons A, Chitnis $\mathrm{X}$ et al. Frontotemporal white matter changes in amyotrophic lateral sclerosis. J Neurol. 2005;252(3):321-31. https://doi.org/10.1007/s00415-005-0646-x

20. Schimrigk SK, Bellenberg B, Schlüter M, Stieltjes B, Drescher R, Rexilius $J$ et al. Diffusion tensor imaging-based fractional anisotropy quantification in the corticospinal tract of patients with amyotrophic lateral sclerosis using a probabilistic mixture model. AJNR Am J Neuroradiol. 2007;28(4):724-30.

21. Sarro L, Agosta F, Canu E, Riva N, Prelle A, Copetti $\mathrm{M}$ et al. Cognitive functions and white matter tract damage in amyotrophic lateral sclerosis: a diffusion tensor tractography study. AJNR Am J Neuroradiol. 2011;32(10):1866-72. https://doi.org/10.3174/ajnr.A2658

22. Agosta F, Ferraro PM, Riva N, Spinelli EG, Chiò A, Canu E et al. Structural brain correlates of cognitive and behavioral impairment in MND. Hum Brain Mapp. 2016;37(4):1614-26. https://doi.org/10.1002/hbm.23124

23. Woolley SC, Strong MJ. Frontotemporal dysfunction and dementia in amyotrophic lateral sclerosis. Neurol Clin. 2015;33(4):787-805. https://doi.org/10.1016/j.ncl.2015.07.011 